\title{
The Study of Public Opinion Guidance and Media Rationality of Mainstream Media in the Media Convergence Environment
}

\author{
Zuowei Zhao \\ Institute of Media \\ Inner Mongolia University for Nationalities \\ Inner Mongolia, China
}

\begin{abstract}
With the continuous development of the Internet and the rapid development of mobile new media in china, media convergence has become the trend of mainstream media. The pattern of information communication has changed greatly, and it has also changed ecological environment of the network public opinion in our country, which has brought new demands to public opinion guidance and media rationality of mainstream media. At present, the network communication has a profound impact on media ecology and the pattern of public opinion in china, the network platform has become "public opinion pool" which in the interest demands and expressions of all sectors of society, emotional catharsis, exchange of ideas. Open, rational, stable sound constitutes a "normal ecological pond", but the network public opinion showed a lower ignition temperature and spread fast, poor credibility etc. So there are some problems such as language violence, group polarization, network siege and other non rational behaviors. In this paper, the causes and manifestations of media irrationality in the public opinion on the social hot events of mainstream media are described, the harm of media irrationality is analyzed and discussed, and the recommendations of media irrational measures are proposed.
\end{abstract}

Keywords-media convergence; mainstream media; public opinion guidance; media rationality

\section{INTRODUCTION}

With the continuous development of network technology and new media, media convergence has become the general trend.From the beginning of 2011, a majority of mainstream media in china has applied their own micro-blog, WeChat, APP and other social medias to disseminate information and actively guide public opinion in the social hot events. The traditional way of spreading information has changed, "everyone has a microphone, everyone is a communicator" has become a reality.

The new media is constructed to be a communication platform for the ordinary people whose public consciousness and citizen consciousness has unprecedentedly enhanced to express their views and release their emotions.

In many social hot events the mainstream media often delivers some irrational viewpoints, so as to guide audiences to express irrational and extreme opinions, leading to frequent public opinion polarization and laceration phenomenon, even to the extent that network abuse, slander, insult, ridicule and other network violence happened, which is not in accordance with journalist professional ethics and norms, and not conducive to building a harmonious network environment of public opinion.

At present, there is few researches on the issue of media rationality in the media convergence environment. Zhi Yunbo (1) analyses suggestions and measures for rational reconstruction of the media from the media culture and mass culture perspective; Xu Yuqing (2) describes the current network hype, individual privacy search and so on media violence in his paper.Yang Xiaohong (3) had focused on one aspect of rational media, but did not reflect media irrationality in the new situation of media convergence social media environment.

One of the theoretical sources of media rationality is Habermas's public sphere theory. Media space is the public domain of contemporary people. In the view of Habermas, the public sphere is the extension of the church and the court, and it is the critical meeting of the political and literary criticism. Because the news communication under the new media environment is more free,media rationality in the traditional sense is facing a new great challenge, and objective and fair principle of media is being eroded in the leading non rational. To analyze the non rational reasons and harms would be the primary purpose of this study.

\section{THE CAUSE OF IRRATIONALITY IN THE GUIDANCE of PUblic OPINION OF THE MAINSTREAM MEDIA}

Under the environment of the media convergence, freedom of speech has reached a high degree,so as to provide the "soil" to the media irrational.On the one hand, due to a variety of the attention economic interests, the new media make the blind pursuit for the net click rates, hits, forwarding capacity and user viscosity.

On the other hand, the sense of professional ethics of media practitioners is declining so that the irrational phenomenon of media is more common in china. How to regulate the bad media behavior is the main content of media self-discipline. 
At the same time, due to lack of renovation of relevant laws and regulations, occupation specification which should timely follow up with the media evolution, the punishment degree of the media anomie is not high, which is caused by one of the reasons for the increased phenomenon of the anomie of media.

\section{THE IRRATIONAL PERFORMANCE OF THE} MAINSTREAM MEDIA IN THE GUIDANCE OF PUBLIC OPINION

The new media is favored by people because of its characteristics of virtual, instant communication, multimedia, participation, equality and freedom.Unfortunately, in recent years there have been a variety of chaotic phenomenon and irrational in media space. Such as all kinds of network bad news are well popular, discourse ethics and language violence shift in micro-blog, WeChat, online community, and other social media, and so gross violations of privacy of public figure by media;The excessive media coverage of controversial topics and biased reportage; New media on social risk events and non rational communication and reporting of news events, parties of moral judgment.

\section{A. First, for the Exclusive News, Mainstream Media}

Abandons the News Professional Standards, Leading to

Public Opinion Reversal. Reversal News Has Become A

Hot Topic in the Field of Chinese Journalism

Under the current media ecology, the rapid development of new media brings media anxiety to traditional media.In the fierce competition of the media, some media blindly grab the news, for fear of losing the "first opportunities"for news reports.In a timely and real options, some medias abandon the news professional standards such as news reality and reportage neutrality.

B. Second, Consciously Catering to the Audience Curious Psychology, Mainstream Medias Highlight the Opposing Factors of the News, and Create Sensational Spread Effects

In addition to rush to send the news, The mainstream medias are also accustomed to express emotional and extreme views on events in the first time, consciously cater to the audience curiosity, in the dissemination of news intentionally highlight the opposing factors to achieve horror, dramatic spread effect.

\section{Third, "Stereotype" to the Audience by Reporting A \\ Group of People Tagged by the Mainstream Media}

The audience often determine the nature of the event itself by stereotype social impression which is by the mainstream media under fragment-information conditions of new media. This media environment is often named "Pseudo Environment".The news literacy of China's Internet users is generally low, and they are lack of independent thinking spirit in the new media public opinion field, so that symbolic-labels communication will be more used and concluded the judgment from the one-sided by cyber citizens .

\section{The Fourth,the Medias Put on the Subjective Presupposition To Report the dispute Facts, and Bring to Bear on "Public Opinion Violence" or "Irrational Solidarity"to One of the Parties}

In the public opinion hot event reporting, the media reporters are easily influenced by personal subjective feelings and cognitive bias, so that news reports are not objective and fair. The medias used to stand high ground in the public opinion, put the premarital determining of the nature,subjective views and position presets to the debatable events.All these behaviors are actually a "public opinion violence", with the media right to bind the audience the right to speak,and to guide the audience on the facts of the error or one-sided understanding.

\section{E. Improper Dissemination of Risk Events}

Due to the huge news value of the risk event itself, it is easy to be concerned by the audience, and often becomes the object of media coverage. In the risk event reports, media will usually cause the risk event propagation distortion for their own professional knowledge, or for the subjective purpose of the media reports through the information deletion improper naturally or half unconsciously, inappropriate information processing, as well as improper use of the specific professional terminology in the specific field.

Especially in some economic reports, this improper performances easily lead to economic losses and social panic. In addition, in a number of negative public opinion events, such as official corruption, conflicts between the government and the people, the contradiction between doctors and patients, the mainstream medias are often exaggerated, blindly follow the trend to report, boost risk events negative public opinion dissemination.

\section{F. Improper Checks to the Network Rumors Spread by the Mainstream Media}

The new media provides an excellent platform for Internet Rumors spread. Because of the network anonymity and the concealment of information dissemination, network rumor trace ability and governance will be difficult.The mainstream medias are usually partial to listen to network media unverified information and reprint it, easily lead to the rapid spread of rumors.

\section{THE IRRATIONAL HARM IN THE GUIDANCE OF PUBLIC OPINION OF THE MAINSTREAM MEDIA}

\section{A. The Media Irrationality can Initiate Frequent Media Public Opinion Reversal Events, and Cause Public Opinion "out of Focus"}

In the event of a hot public opinion,the discussion of public issues of the event itself will be squeezed,so that network space is full of useless information,resulting in serious information pollution and deterioration of public opinion environment and network environment. In the part of the reverse news events, some irrational behaviors and network violence involved such as network slander and abuse,bring the negative effect to the social order and social 
ethics, leading to the deterioration of environment of network public opinion.

\section{B. The Media Irrationality can Bring Erosion and Damage to the Credibility and Authority of the Media}

Media irrational behavior can bring the consequence of serious damage to the credibility of the media.The media authority will be weakened and the public will lose trust in the media for the lack of the media professionalism.

\section{The Media Irrational Behavior will Influence the Objective and Fair of News Reports}

The irrational behavior with strong media subjective emotion will affect the objectivity and impartiality of the media coverage, and the journalists will bring their subjective emotions to the report, which is bound to affect the appearance of the facts.

\section{The Media Irrational Behavior will Influence the Audience's Reception of Valuable Information}

In order to cater to the taste of the audience and attract attention, mainstream medias spread a large number of vulgar, worthless information, not only affecting the audience to accept ,but wasting the media resources.

\section{E. The Media Irrational Behavior may Touch off the Society Disorder and Economic Chaos}

The media irrational behavior will increase the spread of social risk events and the social public events, resulting in economic losses and social unrest, affecting the social and economic order and social stability.

\section{Measures to AVOID the Media IrRationaL BEHAVIOR}

In the environment of media convergence, facing media irrational challenges from the rapid development of the media, from the perspective of media criticism, traditional media and media people should cultivate rational consciousness, overcome the inherent prejudice, adhere to the rational pursuit and rational practice to guide public opinion and resolve conflicts, promote righteousness, consensus, gradually cultivate public rationality, promote the construction of a rational and peaceful social mentality.

In addition, we should strengthen the education of journalism media practitioners, and perfect journalistic ethics and legal system. Media practitioners should deal with the objective and fair coverage of news events, and abandon the subjective and irrational emotions. The mainstream media should improve their ability to recognize and judge the risk events. Improper media coverage of risk events will affect the public's judgment and identification of risk. Media staff should try to make scientific and accurate reports of risk events by reserving adequate professional knowledge in related fields, and reduce the risk of panic and loss caused by improper media coverage of risk events to a minimum.

The mainstream media should not boost negative public opinion dissemination of the risk events. Rational and prudent risk event reports, especially food safety and other economic livelihood event, will be needed. In the event of risk reports, the media should accurately grasp the degree of risk reporting,and avoid to do high frequency intensive coverage of the same incident in the short term, to avoid social panic caused by media irrational reports.

\section{REFERENCES}

[1] Zhi yunbo."The media rationality reconstruction",China Social Science Daily, November 23,2010.

[2] Xu yuqing."Media violence and the construction of media rationality--Media event analysis in move"search"', Youth Journalist, 2012, $21(10): 55-58$.

[3] Yang xiaohong."The operation of communication mode of media rationality in the media age",Journal of Yunnan University of Finance and Economics,2009,23(2):89-92. 